1978). Of 88 non-herbaceous species found along a 1,300-m elevation gradient, 22 were CAM, three were $\mathrm{C}_{4}$ and the remaining 63 were $C_{3}$. Ten samples were taken at $150-\mathrm{m}$ altitude intervals; density, frequency and area cover values were used to determine an elevation. The main peak in the importance of CAM species was found to occur at low altitude, that of $\mathrm{C}_{3}$ species at higher altitudes and the $\mathrm{C}_{4}$ peak was intermediate between the other two. Eickmeier considers this sequence to reflect both the adaptational significance of the physiological variations and also the respective responses to biotic, competitive factors. For example, the CAM plants are most suited to the low altitude, arid environment, where competitive interplay with other plant species may be regarded as minimal (but see Nature op. cit). $\mathrm{C}_{3}$ plants, he claims are evolutionarily attuned to competitive situations with low abiotic stress; $C$ plants can cope with both, having potentially high productivity and the importance value for each species at each

capacity to withstand water stress.

The greater competitive success of $C_{3}$ species in higher altitude, more mesic conditions in the tropics has now been demonstrated by Tieszen, Senyimba, Imbamba and Troughton (Oecologia, Berl. 37, 337; 1979) along an altitudinal and moisture gradient in Kenya. They have found that nearly all the grass species of the low altitude grasslands in Kenya are of a $\mathrm{C}_{3}$ photosynthetic type. Some grass tribes, such as Paniceae and Andropogoneae (both exclusively $\mathrm{C}_{4}$ ) were most frequently encountered at intermediate altitudes. The exclusively $\mathrm{C}_{3}$ tribes (such as Festuceae, Aveneae and Agrostideae) were found only at high altitude. No $\mathrm{C}_{3}$ species were found below an altitude of $2,000 \mathrm{~m}$ and no $\mathrm{C}_{4}$ species above $3,000 \mathrm{~m}$.

Evidently the complex adaptational significance of $\mathrm{C}_{3}, \mathrm{C}_{4}$ and CAM photosynthetic systems is profoundly influential on the floristic composition of plant communities both on a biogeographic and an ecological scale.

\section{Viral pathogenesis and virulence}

\section{from D.A.J. Tyrrell}

IT is easy to say "The influenza virus causes influenza", while having really no scientific explanation of many of the signs and symptoms of the disease, for instance the headache or the depressed white blood cell count. This meeting* would have jolted anyone out of such a complacent view and also gave some good examples of how patient, thoughtful research is building a more complete picture of what happens in some virus infections - how the virus enters and spreads through the body, enters cells, disturbs their function, interacts with the host defence mechanisms (such as interferon or humoral or cellular immunity) and disturbs normal body functions to produce what we recognise as a clinical disease.

What seem in the laboratory to be very similar viruses may nevertheless produce either different diseases or similar diseases by different methods. There was for instance a session on coronaviruses, which all look the same and are similar in biochemical composition. Yet the different coronaviruses are specific to particular species - mouse coronaviruses never seem to affect man or vice versa, for example and sometimes even to a very limited variety of cells in one species. P.A. Bachmann (World Health Organization, Munich) described how the coronavirus of transmissible gastroenteritis of pigs (TGE) affects only the differentiated enterocytes of the villi of the small intestine which may almost disappear. The crypt cells, * A symposium on Mechanisms of Viral Pathogenesis and organised by P.A. Bachmann. however, survive and migrate up to cover the villi again, but while they are doing this and differentiating into mature enterocytes, there are profound physiological disturbances - lack of absorption, excess secretion, lack of digestive function (for instance the splitting of lactose by disaccharidase) leading to marked diarrhoea, which can kill a piglet from dehydration unless it is treated. On the other hand, another pig coronavirus causes vomiting and wasting disease. M. Pensaert and K.L. Andries (State University of Ghent) described how this virus affects the upper respiratory tract and lung, the tonsil and the jejunum; it affects the cells of the plexus of Auerbach and Meissner in the gut and seems to spread from them. It spreads through the nervous system to the brain stem and later to the spinal cord and the rest of the brain. All this was established in great detail by painstaking serial virus assay and immunofluorescent microscopy of experimentally infected animals. This suggests how the disease develops - probably the virus spreads from the respiratory tract and gut along peripheral nerves to the brain stem where the lesions give rise to vomiting, while the damage to neurones in the plexuses prevents normal peristalsis, leading to wasting. Furthermore, knowing that the virus invades the nervous system widely, it is not surprising that a virtually identical virus has been recovered from pigs diagnosed as having encephalitis, and so was named haemagglutinating encephalitis virus.

Explanations of these important and mysterious tropisms of viruses must lie at the molecular level, in the functioning of the peptides and nucleic acids of which viruses are made. B. Fields (Harvard Medical School) gave an example of what can be done, when he described his study of two pairs of genes, represented by the RNAs $\mathrm{S} 1$ and M 2 of reoviruses types 1 and 3 . He studied parent viruses and a collection of recombinants (or reassortants) and showed that S1 nucleic acid specifies the haemagglutinin protein of the virus and that this is responsible for the ability to agglutinate red cells and for serological specifity. It is also responsible for cell tropism in the animal, so that the type 3 virus causes a necrotising encephalitis due to a specific attack on the neurones. It also determines the extent to which the virus inhibits DNA synthesis when absorbed to cells. M2 RNA on the other hand, which is abundant on the surface of the virus particle determines whether the virus resists inactivation by chymotrypsin. M2 RNA from type 3 makes the virus resistant to host enzymes and hence able to infect when given by mouth. Discussion of this work showed that the next step was to determine at which site on the surface of the neurone the virus attached. This is probably a part of the chemical matrix which has some other physiological function characteristic of neurones. Reassortants were also made which were infectious when given by mouth, like type 1 , and caused central nervous system disease, like type 3 , and so, by the oral route, were more pathogenic than either parent. This type of manipulation confirms earlier conclusions about the functions of the genes.

It is very difficult to evaluate the way in which the host defences interact with the virus, sometimes preventing its multiplication, sometimes causing immunological damage to infected tissue. For instance, P.A. Neighbour and B.R. Bloom (Albert Einstein College of Medicine, New York) described some of their work which started when they analysed why the lymphocytes of patients with multiple sclerosis show an unusual reactivity to measles virus; they tracked down the fault to a defect in the ability of their lymphocytes to produce type 1 interferon (Neighbour \& Bloom Proc. natn. Acad. Sci. U.S.A. 76, 476; 1979).

The success of the meeting was obvious, and there were many other interesting papers besides those mentioned here, including whole sessions on herpes viruses and rhabdoviruses. There were clear signs that pathogenesis not only holds scientific interest, but may be a source of practically useful knowledge: vaccines and antiviral treatments do not always work - sometimes because we guess too much and know too little about the process by which viruses produce disease.

D.A.J. Tyrrell is in the Division of Communicable Diseases, Clinical Research Centre, Harrow. 